\title{
Research on Energy Recovery Method of Virtual Track Train Based on Working Conditions Segmentation Strategy
}

\author{
CUI Hongming ${ }^{1}$, PENG Anjin ${ }^{2}$, SONG Pengyun ${ }^{3 *}$, SUN Mengmeng ${ }^{1}$ \\ ${ }^{1}$ College of Electronic Information, Southwest Minzu University, Chengdu 610225, China \\ ${ }^{2}$ College of Continuing Education \& Cadre Training Center, Southwest Minzu University, Chengdu 610225, China \\ ${ }^{3}$ College of Electrical Engineering, Southwest Minzu University, Chengdu 610225, China \\ Email: chmgeren@foxmail.com
}

\begin{abstract}
In order to improve the efficiency of energy recovery of hybrid virtual track train, an energy management method based on the strategy of working condition segmentation is proposed under the condition of known line information. This method adjusts the power allocation of power battery and supercapacitor in different power modes of virtual track train. Based on the environment of MATLAB/Simulink, a simulation system for energy recovery of virtual track train is developed. Then this paper carries on the simulation analysis. The simulation results show that the proposed energy management method can achieve more efficient energy recovery under the condition of meeting the train power demand. After optimization, the power output of the train's power battery is reduced by $9.9 \%$, and the recovery rate of the train's braking energy is increased by $9.3 \%$.
\end{abstract}

Keywords: virtual track train, supercapacitor, energy recovery, energy management.

\section{$1 \quad$ Introduction}

With the development of urbanization, there are many disharmonious phenomena in urban traffic. The deterioration of traffic environment, environmental pollution, energy utilization and noise all cause great pressure on the development of cities. City residents have put forward new requirements for convenient car models. It is an effective countermeasure to develop the urban traffic mode, especially the highefficient traffic mode with urban rail transit as the core.

The self-guided tram (SGT-VT) based on virtual track is proposed in this big situation. It is a new road traffic technology which runs according to the rail transit mode. Virtual track train is the product of the integration of modern city tram and emerging automobile industry technology. It mainly includes unmanned driving, multi-section vehicle track tracking, hub motor drive and modern tram advanced concepts and technologies. Virtual track train does not need to lay physical track, it relies on virtual track. Track information, train speed and corner command information to guide the vehicle to run. By using the predictability of the preset track, the track data are acquired by the information acquisition system, and through complete tracking algorithm and motor control system with reliable performance, the intelligent driving of virtual track train can be realized.

The virtual track train has the characteristics of heavy load and high speed, so the hybrid power system with power battery and supercapacitor is generally adopted. As the power system of the virtual track train is composed of several components, the multi-power sources not only increase the train power capacity, but also increase the complexity of the work control of vehicle traction system. In the process of vehicle operation, in order to reduce the energy consumption of the train and improve the finishing efficiency of the power system, it is necessary to reasonably switch the working mode of each power source and reasonably allocate the train demand power. In order to reduce the operation cost of the vehicle, it is necessary to design some reasonable energy recovery management strategies to use supercapacitors to save battery consumption. The corresponding energy management strategy can improve the energy utilization efficiency of virtual track train. Avoid or reduce the following situations: when the train needs to supplement the peak power, the surplus energy of the supercapacitor is insufficient, and when the train needs to absorb braking energy, the supercapacitor has too much energy left. 
Researchers have proposed a number of energy management strategies for hybrid vehicles, mainly divided into the following kinds: energy management strategy based on logic threshold, such as the literature [1-4] which strategy is the advantage of algorithm is relatively simple and easy to implement. The energy management strategy based on fuzzy logic control, such as literature [5-7], is using fuzzy control theory to fuzzy the predefined rules, improving the robustness of the system, and reducing the influence of the system's nonlinearity. Based on optimization of energy management strategies, such as [8-10]. Optimization idea is usually divided into two kinds: offline optimization and online optimization, offline optimization is a method to solve the extreme value of the objective function under the condition of multiple constraint, and online optimization is a method aiming at real-time optimization, but easy to fall into local optimal solution.

Aiming at the virtual track train which route information is known and using supercapacitor as auxiliary energy. This paper put forward a energy recovery method of virtual track train based on working conditions segmentation strategy, and through the study of the dynamics mode of virtual track train, this method is verified.

\section{$2 \quad$ Virtual Track Train System Model}

\subsection{Dynamic Model of Virtual Track Train}

The premise of vehicle motion control is to carry out mathematical mode of vehicle. The more accurate the model is, the more accurate the description of vehicle motion will be, and the more rigorous the subsequent control of vehicles will be. In order to truly reflect the handling and stability performance of the virtual rail train, this paper introduces longitudinal, lateral, yaw, roll and wheel steering angle changes. Since the virtual track train adopts distributed drive, a four-degree-freedom dynamic model is established for the vehicle body, and its longitudinal motion equation is as follows

$$
M a_{x}=F_{x f l}+F_{x f r}+F_{x r l}+F_{x r r}-\frac{1}{2} c_{W} \rho_{L} A v_{x}^{2}
$$

where $M$ stands for vehicle mass, $a_{x}$ is the absolute longitudinal acceleration of the car, $F_{x f l}, F_{x f r}, F_{x r l}, F_{x r r}$ represents the longitudinal forces of the left front wheel, right front wheel, left and right rear wheel, $c_{W}$ is coefficient of drag, $\rho_{L}$ is air density, $A$ is effective cross-sectional area of vehicle, $v_{x}$ is the longitudinal speed of a vehicle.

Lateral motion equation of vehicle

$$
M a_{y}=F_{y f l}+F_{y f r}+F_{y r l}+F_{y r r}
$$

Equation of vehicle roll motion

$$
I_{x x} \stackrel{\square}{\varphi}=m g\left(h_{s}-h_{w}\right) \varphi-m\left(h_{s}-h_{w}\right)\left(v_{y}+v_{x} \gamma\right)-\left(\zeta_{f}+\zeta_{r}\right) \varphi-\left(E_{f}+E_{r}\right) \varphi
$$

where $I_{x x}$ is the moment of inertia of the vehicle around the X-axis, $\varphi$ is the roll angle of the vehicle, $m$ is the sprung mass of a vehicle, $h_{s}$ is the height from the ground at the centre of mass, $h_{w}$ is the height from the ground at the centre of roll, $\zeta_{f}, \zeta_{r}$ are roll damping parameter of the front suspension and rear suspension respectively, $E_{f}, E_{r}$ are roll stiffness parameter of the front suspension and rear suspension respectively.

Equation of vehicle horizontal swing

$$
I_{z z} \quad \gamma=\left(F_{y f l}+F_{y f r}\right) l_{f}-\left(F_{y r l}+F_{y r r}\right) l_{r}+\frac{d_{f}}{2}\left(F_{x f l}+F_{x f r}\right)+\frac{d_{r}}{2}\left(F_{x r l}+F_{x r r}\right)
$$

where $I_{z z}$ is the yawing moment of inertia of the vehicle, $l_{f}$ is the distance from the centre of mass of the vehicle to the front axle, $l_{r}$ is the distance from the centre of mass of the vehicle to the rear axle, $d_{f}$ is the front wheel base of a vehicle, $d_{r}$ is the rear wheelbase of a vehicle. 


\section{$2.2 \quad$ Dynamic Model of Wheel}

In the actual running process of virtual train, ECU (central electronic control unit) needs to accurately obtain real-time status parameters of virtual train tires for calculation, such as tire output force, which is determined by the real-time status of road conditions and characteristics of tires.

The wheel dynamics model mainly simulates the longitudinal motion state of train wheels. In this paper, ignoring the vertical motion state of train, and detailed models are not established for bogies, only the longitudinal motion state of wheels is analysed. According to the traction demand and running speed demand transmitted by the train model, the wheel dynamics model calculates the wheel demand input torque and speed. Computational formula is

$$
\left\{\begin{array}{l}
I_{r} \omega_{o}=T_{d o}-F_{o} R_{d y n} \quad(o=f l, f r, r l, r r) \\
T_{\text {need }}=\left(F_{\text {trane }}-F_{\text {brane }}\right) R_{d y n}+T_{\text {loss }}+T_{\text {iner }} \\
T_{\text {iner }}=\frac{d \omega_{\text {need }}}{d t} I_{r}
\end{array}\right.
$$

where $I_{r}$ is the moment of inertia of the wheel, $\omega_{o}$ is the angular velocity of the wheel, $T_{d o}$ is the torque of the wheel drive motor, $F_{o}$ is the longitudinal force of a tire, $R_{d y n}$ is the rolling radius of the wheel, $T_{n e e d}$ is the desired input torque of the wheel, $F_{\text {trane }}$ is the desired traction of the wheel, $F_{\text {brane }}$ is the desired braking force of the wheel, $T_{\text {loss }}$ is the loss of wheel torque, $T_{\text {iner }}$ is the accelerating inertia torque of the wheel.

\section{$2.3 \quad$ Hybrid System Structure}

The power system structure of the supercapacitor/power battery virtual rail train studied in this paper is shown in Figure 1. The system is mainly composed of supercapacitor, power battery, traction motor, DC converter and traction inverter.

The power batteries provide the main source of energy for trains. As an auxiliary power supply, the supercapacitor is connected to the DC bus by a two-way DC-DC converter to provide auxiliary energy for the train and recover the braking energy. The main features of the hybrid power system are as follows: the input and output power of the supercapacitor and the power battery can be controlled in real time through the DC-DC converter, with good controllability. The auxiliary power can discharge large current, improve the starting, accelerating and climbing performance of the train, and increase the mileage of the train. Under the premise of ensuring the train running performance, the braking energy can be recovered to the maximum capacity and the energy utilization rate can be improved.

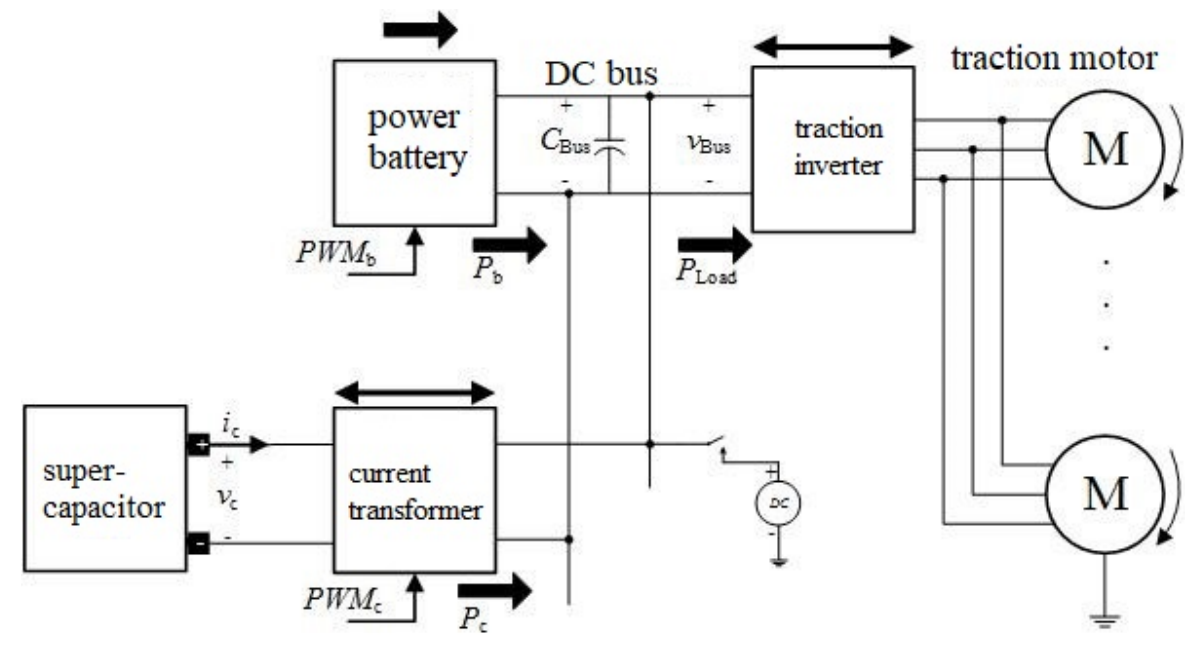

Figure 1. Hybrid system diagram, the figure above shows the dynamic system structure of the virtual track train studied in this paper. 


\section{$3 \quad$ Energy Recovery Strategy Based on System Working mode}

In order to design a reasonable energy management strategy, the working mode of the system under different working conditions should be divided in detail to determine the working scope of each device and set its threshold. The main algorithm ideas of the condition separation strategy proposed in this paper are as follows:

First, the route is divided into several subsections according to the known route information of the virtual tramway, each of which is composed of several low-power modes, several high-power modes and one negative power mode. For each subsection, the off-line energy estimation is carried out according to the demand power with the low power mode as the starting point and the negative power mode as the end point.

Then the energy management strategy of supercapacitor is developed according to the off-line estimation of energy. The energy management system makes reasonable planning according to the motion state of the train and the state of the mixed energy storage system, and controls the mixed energy storage system to work under the optimal state.

Finally, the train will allocate power under the guidance of the energy management strategy. According to the power demand mode and power distribution method, the line is divided into several subsections to estimate and analyse the maximum energy. The reference value of the energy state of the supercapacitor is obtained according to the global optimization algorithm, and the reference value of power distribution is finally determined according to the optimization results of energy management.

\subsection{Line Dividing}

Three power demand modes are defined according to the working mode of the hybrid power system of virtual track train. When the train demand power meets $0 \leq P_{D} \leq P_{E O M}$ ( $P_{E O M}$ is the maximum expected power output of a power cell), the hybrid power system is working in low power mode (LM), when the train demand power meets $P_{D}>P_{E O M}$, the hybrid power system is working in high power mode (HM), when the train demand power meets $P_{D}<0$, the hybrid power system is working negative power mode (NM).

The train running interval is divided into several subsections according to the known route information of the virtual track trolley, and subsections are bounded by points that the negative power mode of the train just ends. First, the set $U$ of the points that train's power mode will change can be defined

$$
\mathrm{U}=\left\{S_{i} \mid P_{D}^{*}\left(S_{i}\right)=0 \& \frac{d P_{D}^{*}\left(S_{i}\right)}{d s}>0\right\}, 0 \leq i \leq n_{1}-1
$$

where $P_{D}^{*}\left(S_{i}\right)$ is the expected power at point $i$, the elements in set $\mathrm{U}$ are arranged in order from smallest to largest.

And the distance between two adjacent positions is defined as a subsection

$$
S S(i)=\left[S_{i}, S_{i+1}\right], 0 \leq i \leq n_{1}-2
$$

where $\left[S_{i}, S_{i+1}\right]$ represents a distance starting with $S_{i}$, ending with $S_{i+1}, n_{1}$ is the number of elements in the set.

According to the above definition, each subsection consists of several low power modes, high power modes, and one negative power mode. The subsection must start with the low power mode and end with the negative power mode.

So, according to the above definition, the set of cut-off points can be divided into three types in more detail. The set of points can be further defined

$$
U_{1}=\left\{S_{j} \mid P_{D}^{*}\left(S_{j}\right) \geq P_{E O M} \& \frac{d P_{D}^{*}\left(\mathrm{~s}_{j}\right)}{d s}>0\right\}, 0 \leq j \leq n_{2}-1
$$


where $U_{1}$ is a set of points that the train switches from low power mode to high power mode, $P_{D}^{*}\left(S_{j}\right)$ is the expected power of the train at station $j, n_{2}$ is the number of elements in the set $U_{1}$.

$$
U_{2}=\left\{S_{k} \mid P_{D}^{*}\left(S_{k}\right) \leq P_{E O M} \& \frac{d P_{D}^{*}\left(\mathrm{~s}_{k}\right)}{d s}>0\right\}, 0 \leq k \leq n_{3}-1
$$

where $U_{2}$ is a set of points that the train switches from high power mode to low power mode, $P_{D}^{*}\left(S_{k}\right)$ is the expected power of the train at station $k, n_{3}$ is the number of elements in the set $U_{2}$.

$$
U_{3}=\left\{S_{l} \mid P_{D}^{*}\left(S_{l}\right)=0 \& \frac{d P_{D}^{*}\left(\mathrm{~s}_{l}\right)}{d s}<0\right\}, 0 \leq l \leq n_{4}-1
$$

where $U_{3}$ is a set of points that the train switches from low power mode to negative power mode, $P_{D}^{*}\left(S_{i}\right)$ is the expected power of the train at station $l, n_{4}$ is the number of elements in the set $U_{3}$.

\subsection{Off-line Energy Estimation Algorithm}

According to Section 3.1, the train power mode in each subsection is fixed. Therefore, the off-line estimation method can be used to calculate the energy consumed by the train in the subsection.

First, when the train is working in high power mode (HM), the high power subsection can be calculated in two parts $\mathrm{H} 1$ and $\mathrm{H} 2$. So, energy consumption type $\mathrm{H} 1$ is defined as $\Delta S O E_{\mathrm{H} 1}$ which is the increase in energy consumption that the train is working in part $P_{\mathrm{D}}^{*}(s)-P_{\mathrm{EOM}}$ relative to supercapacitor SOE (state of energy) when $P_{\mathrm{D}}^{*}(s)>P_{\mathrm{EOM}}$. It means $\mathrm{H} 1$ can calculate the expected energy of the part $P_{\mathrm{D}}^{*}(s)>P_{\mathrm{EOM}}$ in the target power curve when the train is working in high power mode $(\mathrm{HM})$.

$$
\Delta S O E_{\mathrm{H} 1}=\sum_{S=a}^{b} \frac{3.6\left[P_{\mathrm{D}}^{*}(s)-P_{\mathrm{EOM}}\right]}{\eta_{+} E_{\mathrm{Cmax}} V_{\mathrm{t}}^{*}(s)} \Delta s P_{\mathrm{D}}^{*}(s)
$$

where $\eta_{+}$is the energy source output efficiency, $E_{\mathrm{Cmax}}$ is the rated capacity of supercapacitor, $V_{\mathrm{t}}^{*}$ is the target speed, $P_{\mathrm{D}}^{*}(s)$ is the expected power of the train, $a, b$ are the starting and ending positions of section $\mathrm{s}$.

And define the energy consumption type $\mathrm{H} 2$ is defined as $\triangle S O E_{\mathrm{H} 2}$ which is the increase in energy consumption that the train is working in part $P_{\mathrm{D}}^{*}(s) \leq P_{\mathrm{EOM}}$ relative to supercapacitor SOE. It means $\mathrm{H} 2$ can calculate the expected energy of the part $P_{\mathrm{D}}^{*}(s) \leq P_{\mathrm{EOM}}$ in the target power curve when the train is working in high power mode (HM).

$$
\Delta S O E_{\mathrm{H} 2}=\sum_{S=a}^{b} \frac{3.6 P_{\mathrm{EOM}}}{\eta_{+} E_{\mathrm{C} \max } V_{\mathrm{t}}^{*}(s)} \Delta s
$$

Then, when the train is working in negative power mode (NM), the high power subsection can be calculated in two parts N1, N2 and N3. N1 is the energy that can be recovered, N2 is the energy that cannot be recovered on account of too low speed, and N3 is the energy that cannot be recovered on account of overhigh power. In the optimization method in this paper, only the energy of the N1 part is needed.

Define $\triangle S O E_{\mathrm{N} 1}$ is the increment of $\mathrm{N} 1$ to the supercapacitor state of energy (SOE)

$$
\Delta S O E_{\mathrm{N} 1}=\eta_{-} \cdot \sum_{S=d}^{e} \frac{3.6 \max \left(\left|P_{\mathrm{D}}^{*}(s)\right|, P_{\mathrm{EOM}}\right) \operatorname{pos}\left(\left|P_{\mathrm{D}}^{*}(s)\right|-P_{\mathrm{RT}}\right)}{E_{\mathrm{C} \max } V_{\mathrm{t}}^{*}(s)} \Delta s
$$

where

$$
\operatorname{pos}(\text { para })= \begin{cases}1 & \text { para }>0 \\ 0 & \text { para } \leq 0\end{cases}
$$


and $\eta_{-}$is the energy storage system input efficiency, $P_{\mathrm{RT}}$ is the recovery power threshold.

\subsection{Energy Management Strategy}

According to the calculation method of energy consumption in Section 3.2, the reference value $S O E^{*}(S S(i))$ which is supercapacitor's energy state can be obtained in a route subsection. So, if the actual residual energy of the train's supercapacitor as close as possible to the expected, it can greatly improve the energy utilization efficiency of the train. Define actual residual energy $S O E(S S(i))$ to satisfy

$$
S O E(S S(i))=S O E^{*}(S S(i))
$$

In the subsection of the route, there will be multiple stages of energy change. So, the value of $S O E^{*}(S S(i))$ must be determined for different power modes.

In each subsection of the route, before the train needs the supercapacitor to output energy to replenish the power, let the power battery replenish the energy to the supercapacitor until it is full, in this time

$$
\operatorname{SOE}^{*}([a, b])=100 \%
$$

In each subsection of the route, before the train needs the supercapacitor to absorb the braking energy, the energy of the supercapacitor should be used first to leave enough storage space for the braking energy until the energy meets

$$
\operatorname{SOE}^{*}([c, \mathrm{~d}]) \leq \operatorname{SAT}\left(1-\Delta S O E_{N 1}, 40 \%, 100 \%\right)
$$

where $\operatorname{SAT}(p, 40 \%, 100 \%)$ is the saturation constraint operation is performed on the parameter $p$, the lower limit is $40 \%$ and the upper limit is $100 \%$.

And in each subsection of the route, if the train need supercapacitors to supplement power $\left(P_{\mathrm{D}}^{*}(s)>P_{\mathrm{EOM}}\right)$, at such times, the principle of the energy management strategy is to meet the train's demand for power. So, there is no restriction on the value of $S O E^{*}(S S(i))$.

In order to design a reasonable energy management strategy, the working mode of the system under different working conditions should be divided in detail to determine the working scope of each device and set its threshold. For convenience of analysis of the working mode of power balance, define $P_{E}$ is the actual output power battery, $P_{E O M}$ is power battery maximum output power, $P_{D}$ is the expected power, $P_{C}$ is supercapacitor input/output power, $\eta$ is the average efficiency of DC - DC converter. According to the demand power of train traction operation and the power mode of train subinterval, nine power distribution methods of hybrid power system are defined.

M10 mode: this mode is first power allocation method when the train entering $S S(i)$, the power allocation method of state depends on the next point $S_{m}$, power allocation method will go to M20 if point $S_{m}$ satisfies formula

$$
S_{m}>s \quad \& \quad S_{m} \in U_{1} \quad \& \quad\left|S_{m}-s\right|<\left|S_{i i}-s\right|, m<i i<n_{1}-1
$$

where $S_{m}$ is the next cut-off point the train is about to reach, $s$ is the current position of the train. At this time the train is working in low power mode, and the next working mode is high power mode.

But if point $S_{m}$ satisfies formula

$$
S_{m}>s \quad \& \quad S_{m} \in U_{2} \quad \& \quad\left|S_{m}-s\right|<\left|S_{i i}-s\right|, m<i i<n_{2}-1
$$

power allocation method will go to M60, at this time the train to work in low power mode, and the next work mode is negative power mode.

M20 mode: in this mode, the train can switch from low power mode to high power mode. In this mode, since the train runs at low power and the next mode needs auxiliary power from supercapacitor. So, if the supercapacitor is fully charged, the power battery will directly output energy to the motor, and the 
train will enter M30 mode. However, if the supercapacitor is not fully charged, the power battery needs to output the maximum power to supply power to both the motor and supercapacitor at the same time, and the train enters M40 mode. So, in mode M20, the relation between output and input power is formula

$$
\left\{\begin{array}{lr}
P_{E}=P_{E O M}, \quad P_{C}=-\left(P_{E O M}-P_{D}\right) \eta & S O E<100 \% \\
P_{E}=P_{D}, \quad P_{C}=0 & S O E=100 \%
\end{array}\right.
$$

M60 mode: in this mode, the train can change from low power mode to negative power mode. At this time, the train needs to brake with the next cut-off point of route, and recoverable energy will be generated. In this mode, discharge of the supercapacitor will be determined according to the saturation degree of the supercapacitor, so as to leave enough space for braking energy recovery. If the supercapacitor energy meets condition $S O E \leq S A T\left(1-\Delta S O E_{n 1}, 40 \%, 100 \%\right)$, then enter mode M70. But if the supercapacitor energy meets condition $S O E>S A T\left(1-\triangle S O E_{n 1}, 40 \%, 100 \%\right)$, then enter mode M 80 . In M70 mode, the supercapacitor does not supply power to the motor because it has enough space to recover energy. In M80 mode, the remaining space of supercapacitor is too small to recover all the braking energy, so supercapacitor is preferred to supply power to the motor. Its input and output power relation is formula

$$
\begin{cases}P_{E}=P_{D}, \quad P_{C}=0 & S O E \leq S A T\left(1-\square S O E_{n 1}, 40 \%, 100 \%\right) \\ P_{E}=0, \quad P_{C}=P_{D} & S O E>S A T\left(1-\square S O E_{n 1}, 40 \%, 100 \%\right)\end{cases}
$$

M110 mode: in M10 mode, if the real-time demand power of the train exceeds a certain threshold, the strategy will also change. If the demand power of the train is greater than the maximum output power of the power battery $P_{D}>P_{E O M}$, the train will switch to high power mode and the energy management strategy will enter M110 until the power returns to $P_{D} \leq P_{E O M}$. In mode M110, the input and output power relation is

$$
\left\{\begin{array}{l}
P_{E}=P_{E O M} \\
P_{C}=P_{D}-P_{E}
\end{array}\right.
$$

In M10 and M110 modes, if the train's demand power is less than 0, it means that the train switches to negative power mode, and then the energy management strategy enters M100. Its input and output power relation is formula

$$
\left\{\begin{array}{l}
P_{E}=P_{D} \\
P_{C}=0
\end{array}\right.
$$

The control flow of energy management strategy based on the above working pattern is shown in Figure 2. 


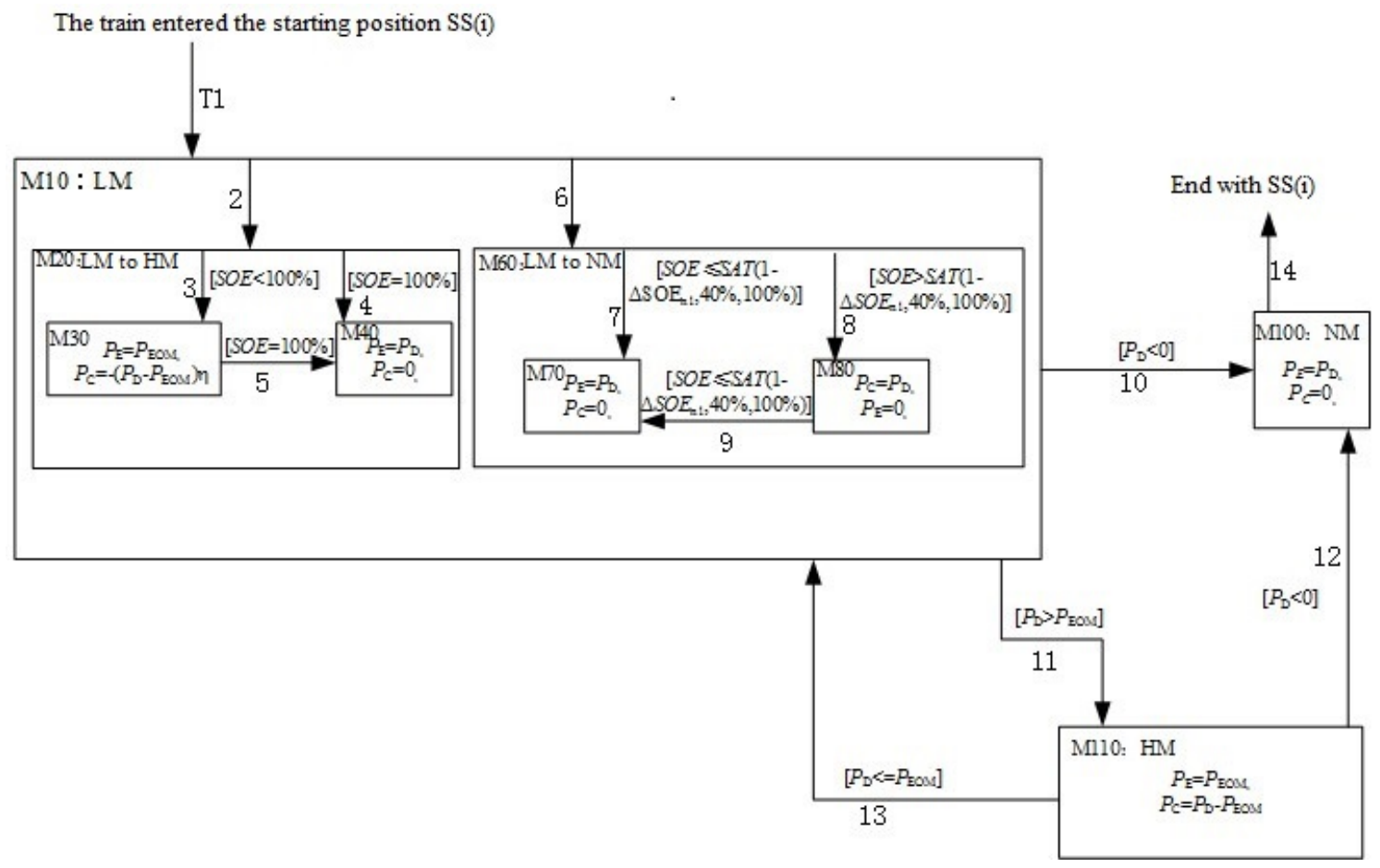

Figure 2. Energy management strategy control flow chart.

\section{Experimental Validation}

\subsection{Simulation Model}

In order to verify the effectiveness of the proposed method, this paper establishes a hybrid virtual rail train simulation model on the MATLAB/Simulink simulation platform based on the energy flow model in Section 1. And the simulation data were compared with the experimental data of a virtual track train under development in Southwest Jiaotong University. Figure 3 is the top block diagram of the system simulation model.

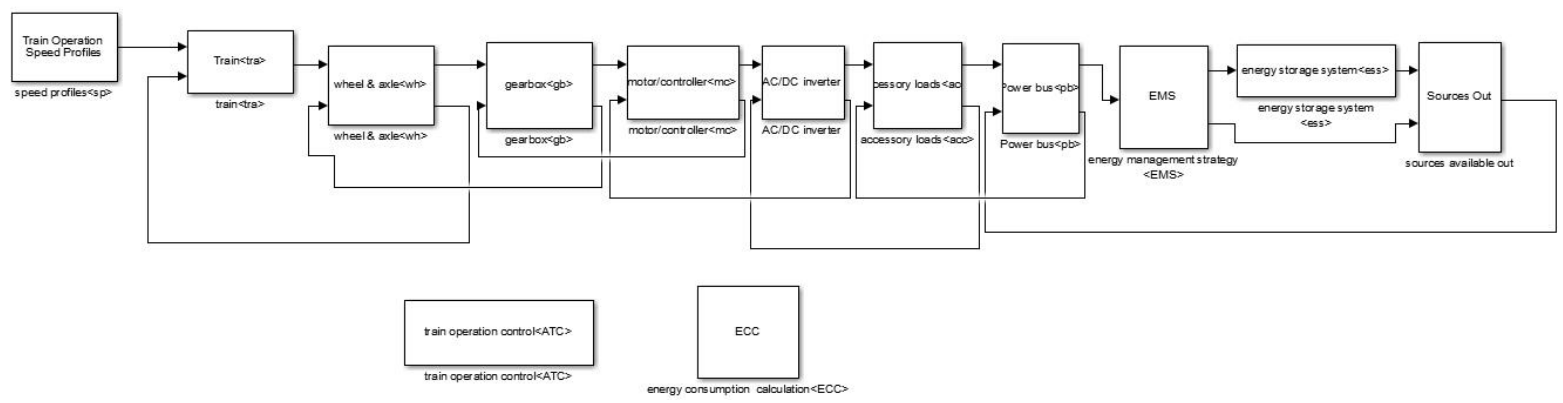

Figure 3. Virtual train simulation model in MATLAB/Simulink.

In this paper, the parameters and line conditions of each model of the simulation system are set to be similar to the test conditions of the sample vehicle, the calculation result curve of power supply energy consumption is obtained as shown in Figure 4. 


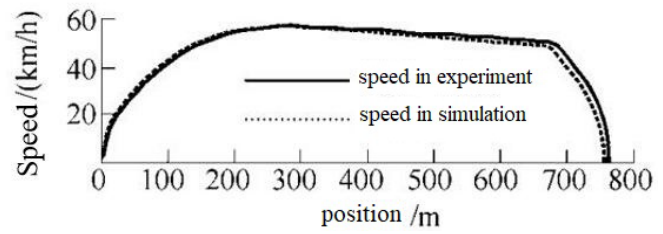

(a)

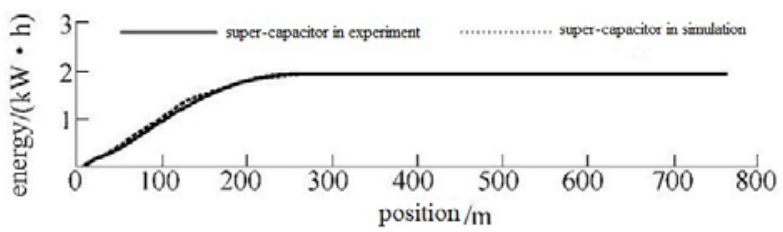

(b)

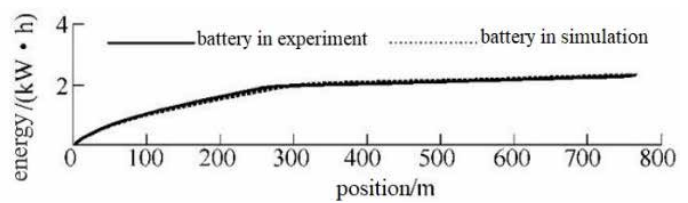

(c)

Figure 4. The comparison curve between the calculated data and the test data of the system simulation model: (a) shows the speed curve of trains running on straight track under line conditions, (b) shows the change comparison curve of energy consumption of supercapacitor, (c) shows the change comparison curve of energy consumption of power battery

According to the comparison and calculation results, compared with the actual vehicle test data, the operating mileage error is $1.8 \%$ and the energy consumption error per kilometre is within $2 \%$ in the running time of 72 seconds. Therefore, it is reasonable for the system simulation model that used in this paper to verify the energy management strategy.

\subsection{Energy Management Strategy Optimization Example}

In order to analyse the effectiveness of the optimization method of energy management strategy in this paper, this chapter takes the virtual rail train described in Section 4.1 of this paper as the prototype for simulation optimization. The route data used in the simulation is a real route.

The main simulation parameters of trains are shown in Table 1.

Table 1. The main parameters of the virtual track train.

\begin{tabular}{ll}
\hline Parameter & Value \\
\hline Train quality & $66 \mathrm{t}$ \\
Register ratio & 6.28 \\
Inertia mass coefficient & 0.09 \\
Basic resistance coefficient A & 2.59 \\
Basic resistance coefficient B & 0.0917 \\
Basic resistance coefficient C & 0.000775 \\
Maximum speed & $60 \mathrm{~km} \cdot \mathrm{h}^{-1}$ \\
Maximum acceleration & $1 \mathrm{~m} \cdot \mathrm{s}^{-2}$ \\
Maximum deceleration & $1 \mathrm{~m} \cdot \mathrm{s}^{-2}$ \\
Auxiliary power consumption & $30 \mathrm{~kW} \cdot \mathrm{h}$ \\
DC-DC efficiency & $92 \%$ \\
\hline
\end{tabular}

The simulation results are as follows: Figure 5 (a) is the goal of simulation to get the train line running speed curve contrast information block division and the curve, it can be seen that in the simulation base according to the preset route and speed of train, figure 5 (b) is the simulation of train target power curve, shown in figure 5 (c) is energy division strategy for trains, in different working condition of interval, the train will be carried out in different power distribution pattern. 


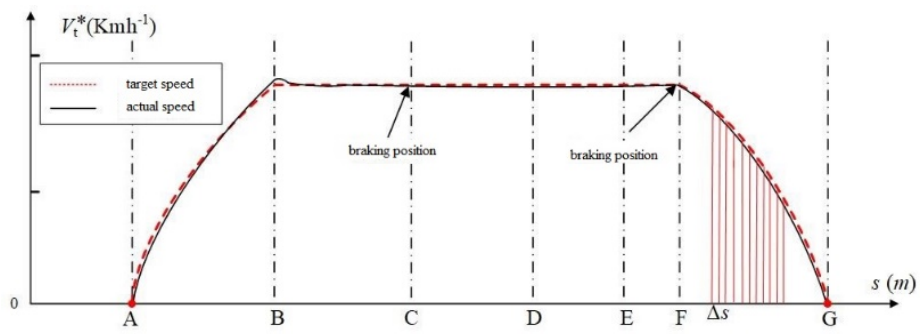

(a)

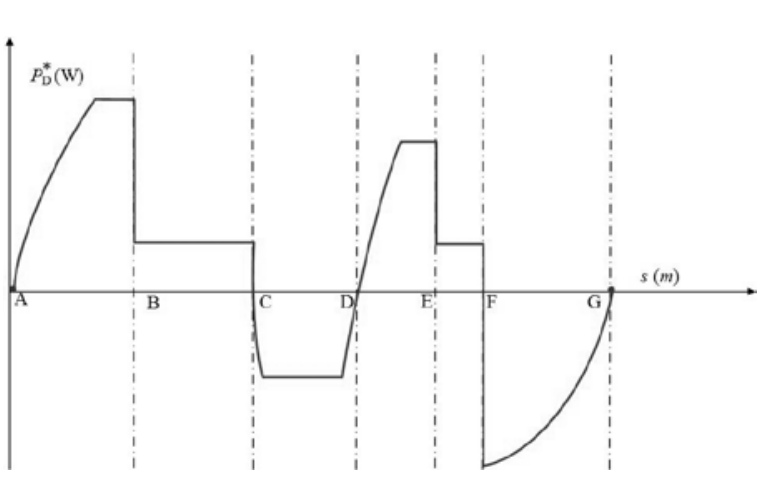

(b)

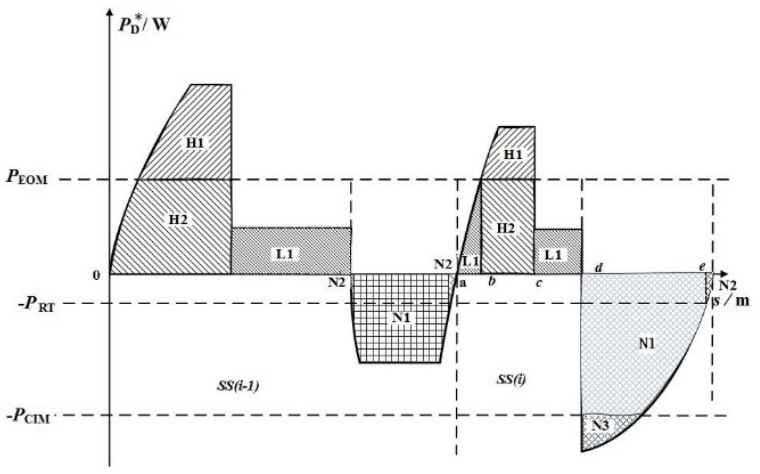

(c)

Figure 5. The simulation result based on working conditions segmentation strategy: (a) is the comparison of target velocity curves, (b) is the simulation of train target power curve, (c) is the result under the guidance of the energy allocation strategy.

The simulation results show that during the simulation, the train has no overspeed and can run automatically according to the predetermined route and speed. The speed acceleration is within the allowable range, and the functional system can also meet the dynamic performance requirements of the train.

This simulation compares the data that used working conditions segmentation strategy (CSS), the data which from the real vehicle and the data that used fuzzy control $(\mathrm{FC})^{[11,12]}$. The optimization results are shown in Table 2 .

Table 2. Optimized result

\begin{tabular}{llll}
\hline Parameter & Real vehicle value & Optimized value with FC & Optimized value with CSS \\
\hline Energy consumption of power battery & $63.0 \mathrm{kWh}$ & $58.5 \mathrm{kWh}$ & $56.8 \mathrm{kWh}$ \\
Energy consumption of supercapacitor & $26.0 \mathrm{kWh}$ & $30.8 \mathrm{kWh}$ & $34.5 \mathrm{kWh}$ \\
Recover energy & $29.55 \mathrm{kWh}$ & $30.4 \mathrm{kWh}$ & $32.2 \mathrm{kWh}$ \\
Total Energy Consumption & $89.0 \mathrm{kWh}$ & $89.3 \mathrm{kWh}$ & $91.3 \mathrm{kWh}$ \\
Average speed of train & $35.73 \mathrm{~km} / \mathrm{h}$ & $35.72 \mathrm{~km} / \mathrm{h}$ & $35.68 \mathrm{~km} / \mathrm{h}$ \\
Running time & $2207 \mathrm{~s}$ & $2207 \mathrm{~s}$ & $2209 \mathrm{~s}$ \\
Recover rate of the breaking energy & $42.3 \%$ & $44.7 \%$ & $51.6 \%$ \\
\hline
\end{tabular}

By comparing the results in Table 2, it can be found that the power battery output of the vehicle is reduced by $9.9 \%$ compared with that before optimization after optimization with CSS. In order to meet the demand of high power, the output of supercapacitor increased by $32.6 \%$ with CSS. This is because according to the requirements of the segmental strategy of working conditions, the train supercapacitor will output for train first before energy recovery, so as to reserve sufficient space to carry the recovered electric energy. After the optimization CSS, the braking energy recovery rate of the train is also increased by $9.3 \%$, which increases the utilization rate of energy. And compared with optimization FC, CSS is more efficient at energy recovery. But the power battery needs high power to power both the 
train and the supercapacitor in CSS, the durability of power batteries will be affected. In conclusion, it can be verified that the segmented strategy proposed in this paper is effective.

\section{Conclusion}

This paper proposes an energy recovery method of virtual track train based on working conditions segmentation strategy, virtual track train by train operation routes according to predetermined rules to block division, and then, train can control power battery and supercapacitor distribution power according to predetermined rules of power, improving the recovery rate of the train braking energy.

Then in this paper, the simulation model of virtual rail train is established on matlab/ Simulink platform and the simulation experiment is carried out. On the premise that the train dynamic performance can be satisfied, the energy recovery strategy is introduced into the model. Simulation results show that the optimized train energy recovery rate increased by $9.3 \%$, power battery energy consumption was reduced by $9.9 \%$, the capacitance of the output power increased by $32.6 \%$, to prove the effectiveness of the recovery strategy. This paper provides some theoretical methods and references for the study of energy management and energy recovery of virtual rail trains.

Acknowledgements. The project was funded by the Southwest Minzu University Graduate Innovative Research Project (Master program CX2020SZ84).

\section{References}

1. Chen Yanqiu, ZHANG Jiye, SONG Pengyun, et al, "Research on operation control of hybrid electric train" Journal of the China Track Society, 2013, 35(8):22-28.

2. YANG Jibin, ZHANG Jiye, SONG Pengyun, et al, "Control strategy of hybrid train power system" Journal of Southwest Jiaotong University, 2015, 50(1):20-26.

3. García P, Fernández L M, García C A, et al. Fuel cell-battery hybrid system integrating two DC/DC converters for transport applications $[\mathrm{C}] / /$ International Conference on Power Electronics and Drive Systems. Taipei: IEEE, 2009:139-143.

4. Herrera V I, Gaztanaga H, Milo A,et al. Optimal operation mode control and sizing of a battery-supercapacitor based tramway[C]// Vehicle Power and Propulsion Conference (VPPC). Montreal Q.C.:IEEE,2015:1-6.

5. WU Jie, WANG Qingyuan, DU Xin, et al, "Control strategy of hybrid train power system" Computer Simulation, 2016, 33(1):161-165.

6. SUN Mengmeng, YANG Jibin, PENG Anjin,, et al, "Research on Energy Management Strategy of Hybrid Tramway Based on Double Fuzzy Logic Control" International Journal of Power and Energy Research, 2019, $3(2)$

7. Chen Lidan, Ou Yanghuilin, "A fuzzy logic control strategy for ev charging and discharging considering vehicle emergency", Electric Power Automation Equipment, 2020, 40(05):62-74.

8. Han Zhang, et al. "Optimal energy management of a fuel cell-battery-supercapacitor-powered hybrid tramway using a multi-objective approach." Proceedings of the Institution of Mechanical Engineers, Part F: Journal of Rail and Rapid Transit 234.5(2020)

9. LI Qi, HUANG Wenqiang, SHANG Weilin, et al, "Fuel Cell Hybrid Tram Braking Energy Recovery Method Based on PMP Energy Management Strategy and Working Condition Piecewise Strategy" Journal of Southwest Jiaotong University, 1-9[2020-10-20].

10. YANG Jibin, XU Xiaohui, ZHANG Jiye, et al, "Multi-objective Optimization of Energy Management Strategy for Fuel Cell Tram" Journal of Mechanical Engineering, 2018,54(22):153-159.

11. HAN Peng, "Research On the Control Strategy of Self-guided Tramcar Based On Virtual Track".2017. Southwest Jiaotong University, $\mathrm{PhD}$ dissertation.

12. YANG Jibin, "Research On Energy Management of Multi-energy Hybrid Electric Tram" Journal of Mechanical Engineering, 2018, Southwest Jiaotong University, $\mathrm{PhD}$ dissertation. 